\title{
Improving thiamine prescribing at an academic hospital network using the computerized provider order entry system: a cohort study
}

\author{
Gregory S. Day MD MSc, Safiya Ladak BScPhm, C. Martin del Campo MD
}

Abstract

Background: Oral thiamine therapy is frequently prescribed to patients at risk for thiamine deficiency despite recommendations emphasizing the need for high doses of parenteral thiamine to reverse brain thiamine deficits. We evaluated the effect of changes to the computerized provider order entry system on the proportion of prescriptions for parenteral thiamine treatment (primary outcome) and dosages prescribed (secondary outcome) within our academic hospital network.

Methods: We obtained data from the pharmacy information system recording thiamine prescribed to inpatients at University Health Network hospitals (Toronto, Ontario) before (Jan. 1, 2010, to Dec. 31, 2011) and after (Nov. 21, 2013, to Apr. 30, 2017) changes to the computerized provider order entry system promoting the use of higher dosages ( $\geq 200 \mathrm{mg})$ of parenterally administered thiamine. Patients receiving thiamine as part of total parenteral nutrition were excluded from analyses, as thiamine prescribing was automated and unlikely to be affected by the intervention.

Results: A total of 6105 thiamine prescriptions were written for 2907 patients before the intervention and 12787 thiamine prescriptions for 8032 patients after the intervention. The proportion of prescriptions for parenteral treatment increased from $55.5 \%$ (3386/6105) to $92.5 \%(11829 / 12787)$ after the intervention $(p<0.001)$. Increases in prescribing of parenteral thiamine treatment were sustained or enhanced across the 3.4-year observation period and were realized across all hospital services. Prescriptions for higher dosages of thiamine increased from $1.1 \%(65 / 6105)$ to $61.4 \%(7845 / 12787)$ after the intervention $(p<0.001)$.

Interpretation: Changes to the computerized provider order entry system were associated with sustained increases in the proportion of prescriptions for high-dose parenteral thiamine therapy. Similar approaches may be leveraged to align prescriber behaviour with well-accepted practice parameters in other areas of medicine.

T npatients are at high risk for thiamine deficiency owing to a preponderance of risk factors, including poor nutritional intake, increased metabolic demand, resuscitation with glucose-containing fluids and medical conditions that impair thiamine absorption from dietary sources. ${ }^{1-6}$ Accordingly, acute thiamine deficiency is commonly encountered in inpatient settings, where it may lead to death in $20 \%$ of untreated or undertreated patients. ${ }^{7,8}$ In historical case series, acute thiamine deficiency led to substantial morbidity, including Wernicke encephalopathy and Korsakoff syndrome, in more than $85 \%$ of survivors. ${ }^{9-11}$ Although the minimally sufficient dosage of parenterally administered thiamine required to correct deficiencies is unknown, dosages in excess of $200 \mathrm{mg}$ are commonly recommended to rapidly reverse brain thiamine deficiency. ${ }^{12-16}$

Despite these recommendations, observational studies consistently report low rates of prescribing of parenteral treatment within academic hospitals. ${ }^{1,9,17,18}$ When parenteral thiamine therapy is prescribed, the dosages and duration of treatment are often below those recommended, ${ }^{1,18-20}$ exposing vulnerable patients to risks of irreversible brain injury and death. ${ }^{7,8,21,22} \mathrm{~A}$ prior attempt to alter prescribing practices using a cost- and effort-intensive strategy (direct pharmacist intervention) failed to alter prescriber behaviour substantially. ${ }^{23}$ Similarly, low-cost efforts (e.g., published guidelines and hospital-wide protocols promoting prescribing of parenteral treatment) exhibit only modest effects on prescribing. ${ }^{1,17,24-26}$ Efficacious means of reversing deficiencies in prescribing are needed.

A review of thiamine prescribing at university-affiliated Canadian tertiary care centres showed low rates of prescribing of parenteral therapy across the majority of hospital services at most of the participating hospitals, including our own. ${ }^{1}$ In

Competing interests: Gregory Day reports personal fees from DynaMed (EBSCO Health), and grants and personal fees from Parabon NanoLabs outside the submitted work. He has provided record review and expert medical testimony on legal cases pertaining to management of Wernicke encephalopathy. He owns stocks (> \$10 000) in ANI Pharmaceuticals. No other competing interests were declared.

This article has been peer reviewed.

Correspondence to: Gregory Day, day.gregory@mayo.edu CMAJ Open 2020. DOI:10.9778/cmajo.20200029 
response to these findings, thiamine prescribing practices were reviewed at University Health Network hospitals (university-affiliated hospitals in downtown Toronto, Ontario), and a system-wide change to computerized order sets favouring prescribing of parenteral thiamine therapy was made. In this way, we leveraged strategies honed in acute care $^{27}$ and community-based settings ${ }^{28}$ to promote adequate treatment of inpatients with suspected thiamine deficiency. We evaluated the effect of these changes on the overall rates of prescribing of parenteral thiamine therapy (primary outcome) and dosages of thiamine prescribed (secondary outcome) at our tertiary care centre.

\section{Methods}

\section{Setting}

This study was conducted at 2 University Health Network hospitals (Toronto General Hospital and Toronto Western Hospital). Data were obtained from the computerized provider order entry system (Centricity, GE Healthcare) recording thiamine prescriptions processed by the centralized hospital pharmacy before (Jan. 1, 2010, to Dec. 31, 2011) and after (Nov. 21, 2013, to Apr. 30, 2017) the system-wide change to computerized order sets (intervention). Preintervention data were collected under a separate protocol permitting retrospective evaluation of thiamine prescriptions. ${ }^{1}$ Data were not available from Jan. 1, 2012, to Nov. 20, 2013.

\section{Study design and recruitment}

Following internal review of preintervention data (see data for University Health Network hospitals, Hospital 6A, in our prior publication ${ }^{1}$ ), changes emphasizing administration of high dosages of thiamine administered parenterally to patients at risk were proposed (Appendix 1, Supplemental Figure S2, available at www.cmajopen.ca/content/8/2/E383/suppl/DC1), approved and implemented within the computerized order entry system (Figure 1). Approval was communicated to hospital staff via a medical bulletin on Nov. 28, 2013, describing the changes and referencing publications supporting the diagnosis ${ }^{29}$ and treatment of patients at risk for thiamine deficiency. ${ }^{14,15}$ Additional details concerning the intervention are included in Appendix 1.

Anonymized thiamine prescribing data were recorded prospectively from Nov. 21, 2013, to Apr. 30, 2017. Briefly, patients were assigned a random study number linked to prescription information specifying the dosage of thiamine, route of administration (oral [per os, nasogastric tube, orogastric tube, gastric tube] v. parenteral [intravenous, intramuscular]), frequency of dosing (e.g., daily, twice daily, 3 times daily), start and end date of thiamine treatment, prescribing physician and inpatient location. We simplified prescriber subspecialty designations to emergency department, intensive care unit (including medical, surgical and trauma units), medical subspecialty (e.g., cardiology, endocrinology, gastroenterology, medical oncology, rheumatology), general internal medicine, neurology, psychiatry and surgical services (e.g., general surgery, cardiac surgery, neurosurgery, orthopedics, gynecol- ogy). Patients receiving thiamine as part of total parenteral nutrition were excluded from the pre- and postintervention data sets, as thiamine prescribing was automated and unlikely to be affected by the intervention.

We measured the total number of prescriptions, number of first prescriptions (a proxy measure defining the number of unique patients treated) and number of doses of thiamine prescribed before and after changes were implemented. The accuracy and reliability of the pharmacy information system were assured through regular comprehensive audits conducted by pharmacy staff.

\section{Statistical analysis}

Prescriptions were stratified by route of administration (parenteral v. oral), and annualized prescribing rates were derived by dividing by the number of years of observation to facilitate comparison across pre- and postintervention periods. We calculated proportions by dividing parenteral prescriptions (or first prescriptions, or doses) by total prescriptions (or first prescriptions, or doses). We compared the proportion of parenteral thiamine therapy prescribed before and after the intervention using $\chi^{2}$ tests ( 1 degree of freedom).

We compared changes across hospital services by subtracting the average proportional change in parenteral prescribing observed across all services from the service of interest, and dividing the difference by the population standard deviation, generating a $Z$ score. We inferred $p$ values from the standard normal distribution, where $p=0.05$ corresponds to $Z= \pm 1.96$, and $p=0.01$ to $Z= \pm 2.58$. The magnitude of the association was conveyed with the use of relative risks $( \pm 95 \%$ confidence intervals [CIs]). We used univariate regression to evaluate the relation between prescribing behaviour (dependent variable) and time in months since the intervention. Statistical analyses were performed with SPSS Statistics 24 (IBM Corporation). Significance was defined as $p<0.05$.

\section{Ethics approval}

Study objectives, methods and procedures were approved by the University Health Network Research Ethics Board. A waiver of consent was granted permitting collection and reporting of anonymized data.

\section{Results}

In total, 18892 prescriptions for 36807 doses of thiamine were provided to 10939 patients across the study period. Changes to the computerized provider order entry system promoting prescribing of parenteral thiamine therapy for patients at risk for thiamine deficiency were associated with a substantial shift in prescribing practices. Following the intervention, the proportion of prescriptions for parenteral thiamine therapy increased from $55.5 \%(3386 / 6105)$ to $92.5 \%$ (11 829/12 787) $(p<0.001)$, and the proportion of parenteral doses prescribed increased from $44.2 \%$ (7052/15 947) to 92.8\% (19 357/20 860) $(p<0.001)$. Thiamine was prescribed to $60.7 \%$ more patients following the intervention, increasing from 1454/year to 2336/year $(p<0.001)$. 


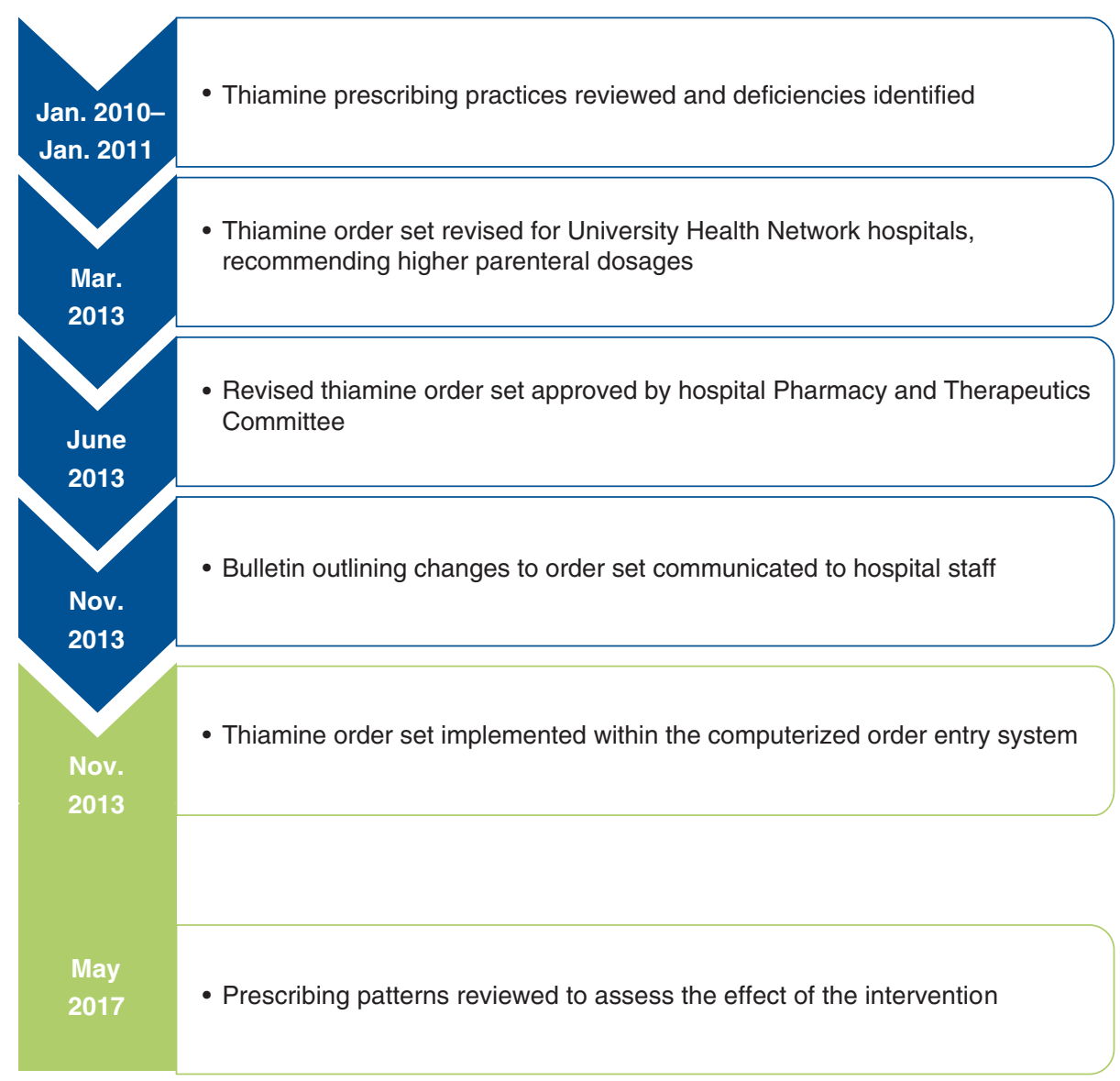

Figure 1: Timeline of review and implementation of changes to the computerized provider order entry system.

Improvements in prescribing of parenteral thiamine therapy were realized across all clinical services (average percentage increase $79.0 \%$ [standard deviation 59.6\%] [range 28.0\%$198.2 \%$ ]) (Table 1). Psychiatry providers showed greater-thanaverage increases in rates of parenteral therapy prescribing, with the proportion of prescriptions increasing from $26.1 \%$ before the intervention to $77.9 \%$ after the intervention $(z=$ $2.0 ; p=0.045)$, although annualized rates of prescribing of parenteral therapy were lower than those observed for other services $(z=-1.98 ; p=0.048)$. Similar relations were observed when the annualized number of doses prescribed was considered. When only first prescriptions were considered, all services achieved rates of parenteral therapy prescribing greater than $80 \%$. Increases in the proportion of parenteral therapy prescribing were matched by increases in the total numbers of prescriptions issued by providers in the emergency department and intensive care unit. The opposite relation was observed with providers affiliated with other services, where propor- tional increases in parenteral therapy prescribing were driven by disproportionate decreases in prescriptions for oral thiamine therapy.

Figure 2 depicts changes in prescribing behaviour after the intervention. The number of total prescriptions for thiamine and doses prescribed remained stable month to month following the intervention, whereas the number of first prescriptions for thiamine increased, on average by 0.83 per month $(95 \%$ CI 0.34 to 1.31 ). The proportion of parenteral therapy prescriptions also remained stable or increased incrementally following the intervention (Table 2).

Changes to the order entry system were also associated with changes in the dosages and schedules prescribed (Figure 3). Before the intervention, prescribers exhibited a near-universal approach to thiamine dosage, with $91.6 \%$ (5592/6105) of prescriptions issued for $100 \mathrm{mg}$ of thiamine. Following the intervention, the proportion of prescriptions for $100 \mathrm{mg}$ of thiamine decreased to $34.9 \%$ (4457/12 785). 
Table 1: Annualized thiamine prescribing before and after changes to the computerized provider order entry system, stratified by service

\begin{tabular}{|c|c|c|c|c|c|c|c|}
\hline \multirow[b]{2}{*}{ Variable; prescribing service } & \multicolumn{2}{|c|}{ Before intervention } & \multicolumn{2}{|c|}{ After intervention } & \multicolumn{2}{|c|}{ Percentage change } & \multirow[b]{2}{*}{$\mathrm{RR}(95 \% \mathrm{Cl})$} \\
\hline & $\begin{array}{c}\text { Total no. of } \\
\text { prescriptions }\end{array}$ & $\begin{array}{c}\% \\
\text { parenteral }\end{array}$ & $\begin{array}{c}\text { Total no. of } \\
\text { prescriptions }\end{array}$ & $\begin{array}{c}\% \\
\text { parenteral }\end{array}$ & $\begin{array}{c}\text { Total no. of } \\
\text { prescriptions }\end{array}$ & $\begin{array}{c}\% \\
\text { parenteral }\end{array}$ & \\
\hline \multicolumn{8}{|c|}{ Annualized no. of prescriptions for thiamine } \\
\hline Emergency department & 675 & 62.7 & 1592 & 87.9 & 135.8 & 40.2 & 1.40 (1.34 to 1.46$)$ \\
\hline Intensive care unit & 181 & 77.3 & 747 & 98.9 & 313.7 & 28.0 & $1.28(1.21$ to 1.35$)$ \\
\hline Medical subspecialty & 309 & 49.9 & 82 & 91.8 & -73.5 & 83.9 & 1.84 (1.69 to 2.01$)$ \\
\hline Medicine & 968 & 46.2 & 752 & 94.5 & -22.3 & 104.7 & 2.05 (1.95 to 2.15$)$ \\
\hline Neurology & 90 & 70.4 & 56 & 93.7 & -37.9 & 33.1 & 1.33 (1.20 to 1.47$)$ \\
\hline Psychiatry & 44 & 26.1 & 20 & 77.9 & -55.1 & 198.2 & 2.98 (2.05 to 4.33$)$ \\
\hline Surgery & 787 & 57.8 & 471 & 95.1 & -40.2 & 64.7 & 1.65 (1.78 to 1.72$)$ \\
\hline \multicolumn{8}{|c|}{ Annualized no. of first prescriptions for thiamine } \\
\hline Emergency department & 320 & 64.5 & 1394 & 88.0 & 335.6 & 36.3 & 1.36 (1.29 to 1.45$)$ \\
\hline Intensive care unit & 97 & 72.5 & 369 & 99.1 & 282.1 & 36.7 & $1.37(1.25$ to 1.49$)$ \\
\hline Medical subspecialty & 145 & 47.2 & 40 & 90.4 & -72.7 & 91.4 & 1.91 (1.68 to 2.19$)$ \\
\hline Medicine & 505 & 45.6 & 251 & 95.0 & -50.3 & 108.4 & 2.08 (1.95 to 2.23$)$ \\
\hline Neurology & 44 & 77.3 & 21 & 97.2 & -53.1 & 25.8 & $1.26(1.12$ to 1.42$)$ \\
\hline Psychiatry & 20 & 25.0 & 11 & 84.6 & -43.3 & 238.5 & 3.38 (1.95 to 5.89$)$ \\
\hline Surgery & 324 & 57.5 & 251 & 96.4 & -22.5 & 67.7 & $1.68(1.57$ to 1.79$)$ \\
\hline \multicolumn{8}{|c|}{ Annualized no. of doses of thiamine prescribed } \\
\hline Emergency department & 1386 & 31.1 & 1683 & 88.5 & 21.5 & 184.5 & 2.84 (2.69 to 3.01$)$ \\
\hline Intensive care unit & 482 & 70.1 & 1340 & 97.9 & 178.0 & 39.5 & 1.39 (1.34 to 1.45$)$ \\
\hline Medical subspecialty & 969 & 40.6 & 189 & 93.2 & -80.5 & 129.6 & 2.30 (2.17 to 2.43$)$ \\
\hline Medicine & 2457 & 43.3 & 1810 & 93.0 & -26.3 & 114.6 & 2.15 (2.08 to 2.22$)$ \\
\hline Neurology & 248 & 67.1 & 129 & 94.1 & -48.1 & 40.2 & $1.40(1.31$ to 1.50$)$ \\
\hline Psychiatry & 167 & 15.9 & 44 & 71.9 & -73.3 & 351.7 & 4.52 (3.46 to 5.89$)$ \\
\hline Surgery & 2266 & 48.8 & 870 & 93.8 & -61.6 & 92.0 & 1.92 (1.86 to 1.98$)$ \\
\hline
\end{tabular}

Conversely, prescriptions for higher dosages of thiamine $(\geq 200 \mathrm{mg}$ ) increased from $1.1 \%(65 / 6105)$ to $61.4 \%$ (7845/ 12 787). Although most thiamine prescriptions continued to be prescribed for 1-time or once-daily dosing $(99.3 \%$ [6060/6100] before intervention, 93.8\% [11 994/12 785] after intervention), prescriptions for thiamine 3 times daily (or more frequently) increased from $0.5 \%(33 / 6100)$ to $6.0 \%$ (774/12 785).

\section{Interpretation}

Changes to the computerized provider order entry system promoting prescribing of parenteral thiamine therapy to patients at risk at our tertiary care hospital were associated with an abrupt increase in parenteral prescribing and the number of patients prescribed thiamine per year. These gains were associated with a decrease in the annualized number of doses of thiamine prescribed, which confirms that clinicians opted for shorter courses of thiamine treatment.
Most encouraging, changes in prescribing behaviours were sustained across the 3.4-year observation period and were realized across all prescribing services. Improvements were also noted in the proportion of prescriptions for higher dosages ( $\geq 200 \mathrm{mg}$ ) of thiamine and, to a lesser degree, the frequency of administration. The magnitude of the association was strongest among prescribers from front-line services, including the emergency department and intensive care unit. These changes may have preempted subsequent prescribing by downstream services, which would account for postintervention decreases in total numbers of thiamine prescriptions issued by providers affiliated with general and subspecialty medical, neurology, psychiatry and surgical services.

Robust changes in prescriber behaviour following the intervention far exceeded the modest benefits commonly attributed to hospital-wide protocols promoting the use of parenteral thiamine therapy in patients at risk..$^{1,17,24-26}$ The observed response also exceeded benefits associated with the use of a clinical decision-support tool promoting prescribing of 
A

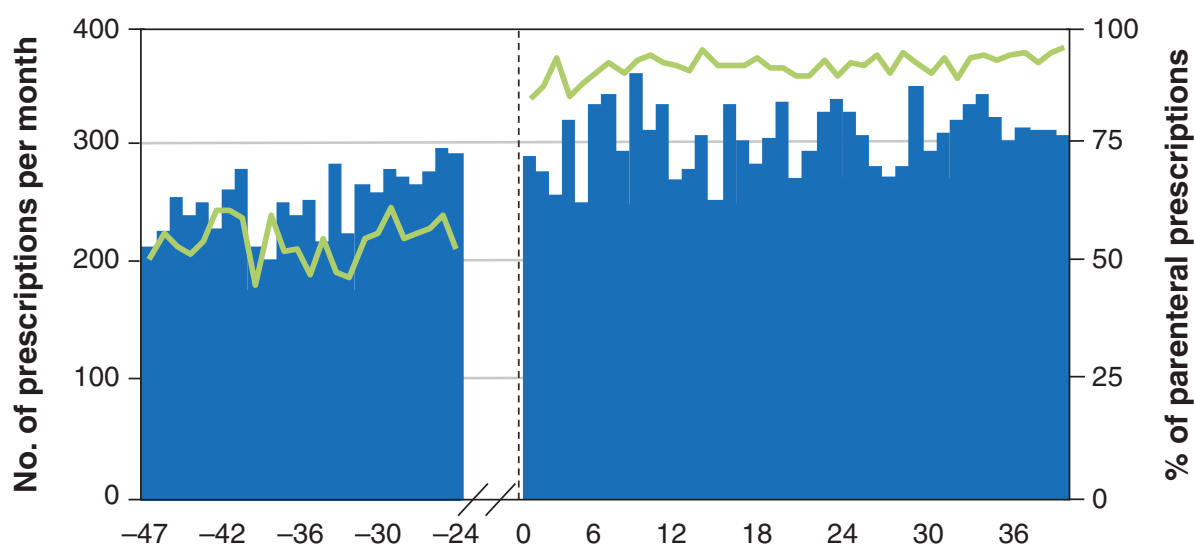

B

Time since intervention, mo

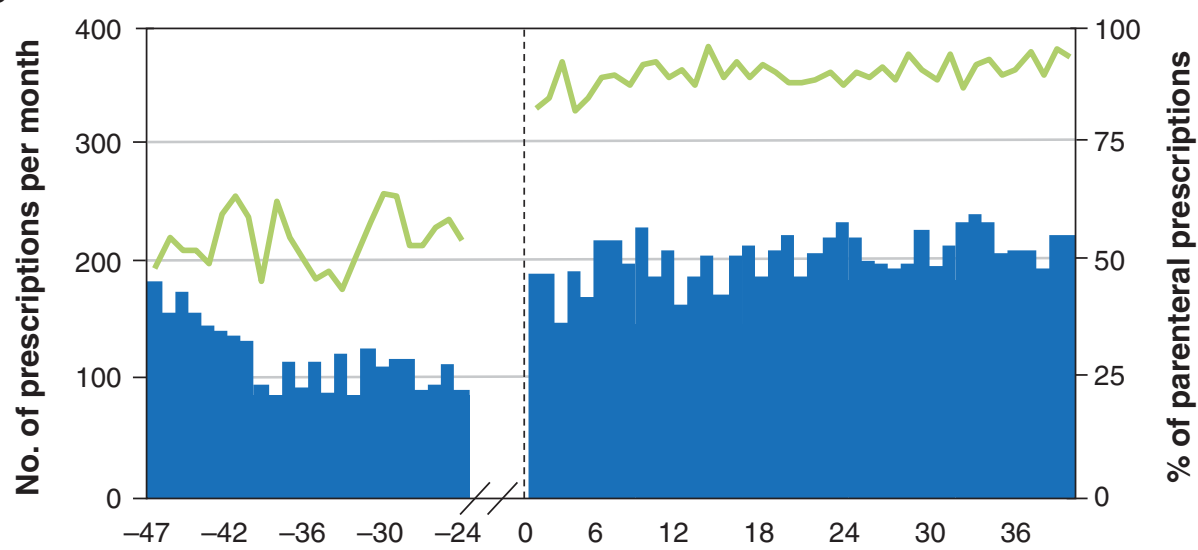

C

Time since intervention, mo

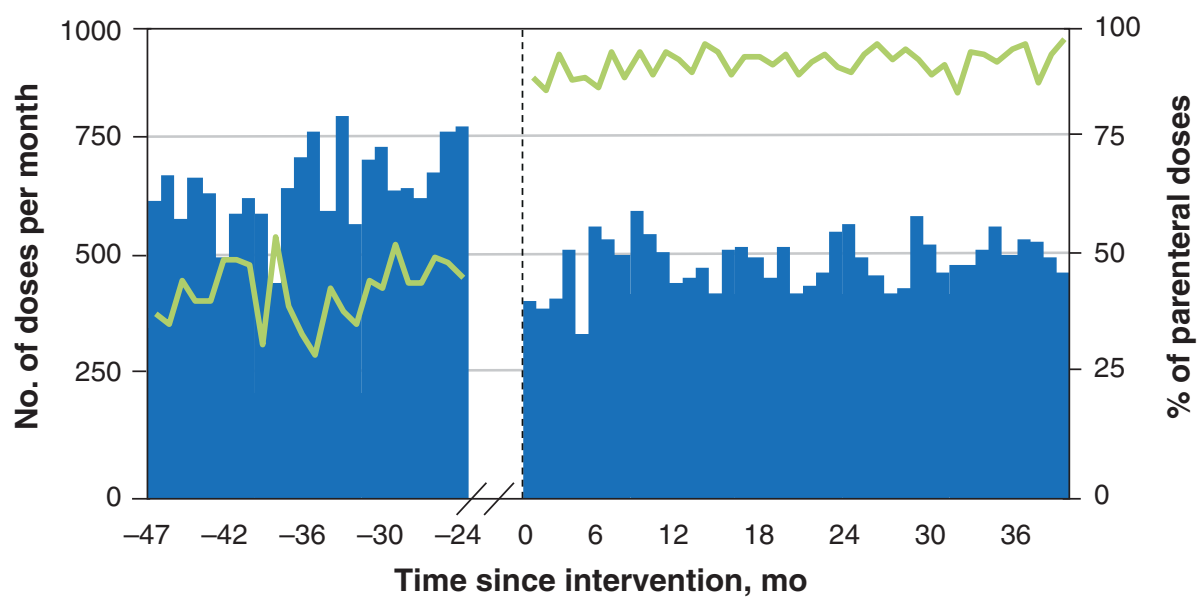

Figure 2: Total thiamine prescriptions $(A)$, first prescriptions for thiamine $(B)$ and doses of thiamine prescribed $(C)$ before and after the intervention (dashed line). The green trend line represents the proportion of prescriptions (A, B) or doses (C) for parenteral thiamine therapy at each time point.

high-dose parenteral thiamine therapy for patients with suspected alcohol use disorder admitted to an urban New York hospital. In that study, implementation of the tool, which auto- populated thiamine order sets in appropriate patients, was associated with an increase in the number of patients receiving appropriate treatment, from $2.7 \%(3 / 113)$ to $20.2 \%(19 / 94) .^{30}$ 


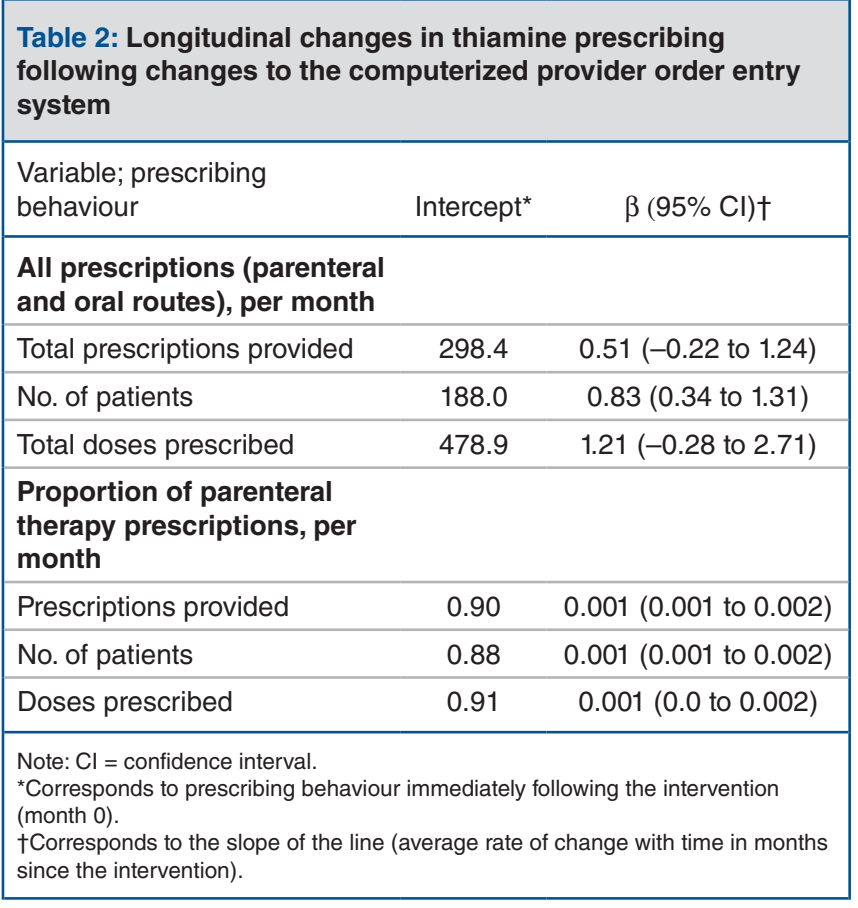

The stronger-than-expected associations in our study may reflect key differences in design and implementation of the intervention. By codifying recommendations as the default selection within order entry systems, our intervention made it easy for prescribers to "do the right thing" and more difficult to deviate from recommended prescribing strategies. In addition, by not tying recommendations to specific clinical diagnoses (e.g., those meeting criteria for alcohol use disorder), we simplified the prescribing process, removing the need for clinicians to identify eligible patients.
We acknowledge that such a simplified approach may not be appropriate in other clinical scenarios. It is critical to carefully weigh the potential risks and benefits associated with any proposed intervention, including potential medication costs and the effort and burden associated with administration, before implementation. This is especially important when considering interventions that may be associated with specific risks or high costs (e.g., pharmacological prophylaxis of venous thromboembolism). In this case, our strategy was justified by the high potential for misdiagnoses and underrecognition of inpatients at risk for thiamine deficiency (particularly those without a history of alcohol use disorder), ${ }^{15,24,31,32}$ the importance of rapid replacement of thiamine in acutely deficient patients, ${ }^{10,13}$ the low risk of adverse effects associated with parenteral administration, ${ }^{33-35}$ and the comparatively high morbidity, ${ }^{9-11}$ mortality ${ }^{7,8}$ and costs $^{36}$ associated with missed treatment or undertreatment of patients at risk.

We leveraged an existing order entry system to implement our intervention efficiently and in a cost-effective manner. This strategy offered compelling advantages over more traditional approaches that rely on labour-intensive pharmacybased interventions and manual chart review, ${ }^{23}$ or educational initiatives, which need to be revised and repeated to keep up with staff turnover and changes in clinical rotations that are especially common in academic hospitals. ${ }^{37}$ Although the use of anonymized data precluded consideration of patient-specific outcomes in this study, the use of standardized order sets in acute care settings has been associated with reductions in hospital length of stay, mortality and medication errors in nonrandomized studies in patients receiving treatment for a wide variety of acute and chronic diseases. ${ }^{27}$

As electronic medical records become increasingly ubiquitous in health care, it will be possible to extend this approach to address other diagnostic and therapeutic shortcomings in

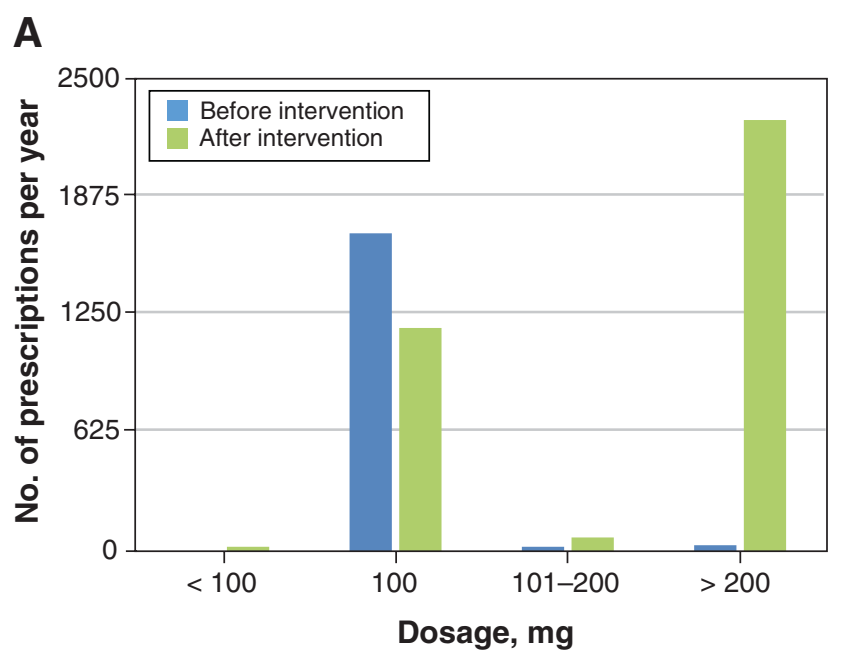

B

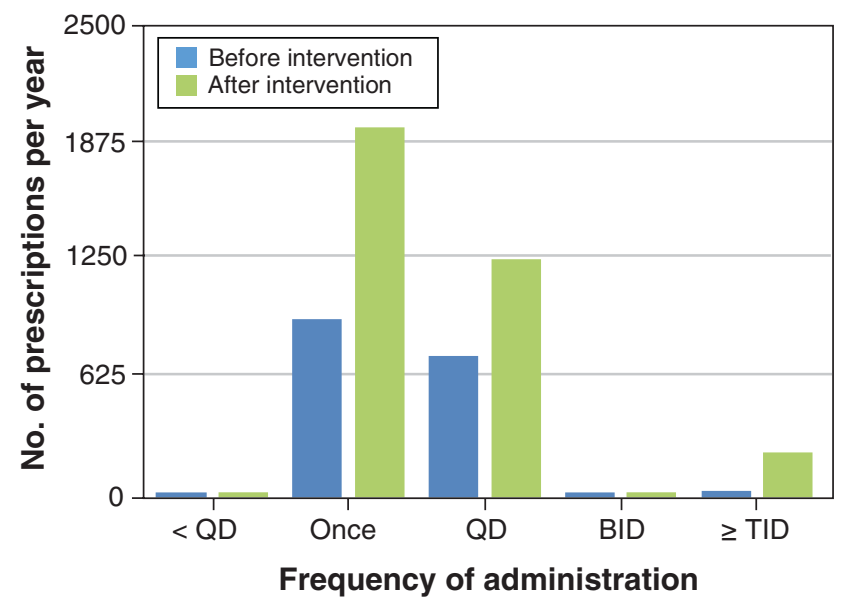

Figure 3: Annualized dosage $(A)$ and frequency of administration $(B)$ of parenteral thiamine therapy prescribed before and after the intervention. Note: $\mathrm{BID}=$ twice daily, $\mathrm{QD}=$ once daily, $\mathrm{TID}=3$ times daily. 
areas of medicine that are supported by well-accepted practice parameters or guidelines (e.g., infection risk reduction in intensive care units, ${ }^{38}$ management of acute exacerbations in patients with chronic obstructive pulmonary disease $\left.{ }^{39}\right)$. With this in mind, it is increasingly important to decipher the factors that influence responsiveness to interventions designed to modify prescriber behaviour, including the degree of consensus concerning the recommended treatment approach, the potential for benefit versus adverse effects associated with the intervention, and the perceived costs and barriers associated with prescribing and administering medications.

It is likely that some of the factors mentioned above contributed to the lower-than-expected rates of prescribing of parenteral treatment among psychiatric prescribers at our hospital. For instance, patients admitted to psychiatry may have declined or resisted intravenous access owing to perceived stigma (this is less common on other services, where intravenous access is universally established), and psychiatric care providers may have encountered additional barriers when attempting to establish or maintain intravenous access outside of medical wards. It is particularly important to explore alternative or additional approaches to optimize prescribing by psychiatric providers for several reasons: acute thiamine deficiency may cause or exacerbate presenting symptoms; ${ }^{8}$ patients with psychiatric illnesses may be at particularly high risk for thiamine deficiency (due to malnutrition associated with eating disorders, substance abuse or somatoform disorders $^{9,18,40,41}$ and may have higher rates of comorbid physical illnesses; ${ }^{42}$ and high-dose parenteral thiamine treatment may correct neurocognitive deficits in patients at high risk. ${ }^{43}$

Efforts are also needed to understand the factors that underlie the strong preference for 1-time or once-daily dosing of thiamine noted within our hospital network and elsewhere. ${ }^{1,19}$ Although most studies to date have considered the impact of a single intervention on prescribing practices, future studies are needed to assess the effect of multifaceted approaches integrating changes to order entry systems together with educational approaches targeting specific services and prescribing patterns.

\section{Limitations}

This study used anonymized data obtained from pharmacy information systems. Thus, it was possible to determine only how much thiamine was prescribed, not what was actually delivered to patients. Similarly, it was not possible to determine whether our intervention improved recognition and treatment of patients at highest risk for thiamine deficiency. By extension, we were unable to consider the specific indications for prescribing - which precluded subanalyses of prescribing behaviour for patients with suspected Wernicke encephalopathy - or whether our intervention led to measurable improvement in patient outcomes. Although routine internal audits supported the accuracy and reliability of the pharmacy information, the lack of peer-reviewed publications supporting the findings of the audits must also be acknowledged.

In addition, this study included data from inpatients within a single hospital network and excluded participants receiving thiamine as part of total parenteral nutrition. Thus, our findings need to be replicated within other hospital environments to establish generalizability and understand how centrespecific factors (e.g., order entry procedures) influence compliance with existing recommendations, responsiveness to changes to order entry systems and treatment in specific patient populations.

Last, unmeasured factors may have contributed to the reported effect, including publication and distribution of articles promoting the use of parenteral thiamine therapy after the intervention. ${ }^{1,13,15}$

\section{Conclusion}

Changes to computerized provider order entry systems were associated with substantial changes in thiamine prescribing behaviours that were sustained across the observation period and realized across all prescribing services within our hospital network. If validated in other populations, changes to order entry systems may be leveraged to modify prescriber behaviour efficiently and in a cost-effective manner, improving compliance with clinical recommendations and patient care.

\section{References}

1. Day GS, Ladak S, Curley K, et al. Thiamine prescribing practices within university-affiliated hospitals: a multicenter retrospective review. 7 Hosp Med 2015;10:246-53

2. Singh H, Watt K, Veitch R, et al. Malnutrition is prevalent in hospitalized medical patients: Are housestaff identifying the malnourished patient? Nutrition 2006;22:350-4.

3. Correia MITD, Waitzberg DL. The impact of malnutrition on morbidity, mortality, length of hospital stay and costs evaluated through a multivariate model analysis. Clin Nutr 2003;22:235-9.

4. Edington J, Boorman J, Durrant ER, et al. Prevalence of malnutrition on admission to four hospitals in England. The Malnutrition Prevalence Group. Clin Nutr 2000;19:191-5.

5. Kirkland LL, Kashiwagi DT, Brantley S, et al. Nutrition in the hospitalized patient. 7 Hosp Med 2013;8:52-8.

6. van Snippenburg W, Reijnders MGJ, Hofhuis JGM, et al. Thiamine levels during intensive insulin therapy in critically ill patients. 7 Intensive Care Med 2017;32:559-64.

7. Harper C. Wernicke's encephalopathy: a more common disease than realised. A neuropathological study of 51 cases. 7 Neurol Neurosurg Psychiatry 1979;42: 226-31.

8. Harper CG, Giles M, Finlay-Jones R. Clinical signs in the WernickeKorsakoff complex: a retrospective analysis of 131 cases diagnosed at necropsy. 7 Neurol Neurosurg Psychiatry 1986;49:341-5.

9. Cook CC, Hallwood PM, Thomson ADB. Vitamin deficiency and neuropsychiatric syndromes in alcohol misuse. Alcobol Alcobol 1998;33:317-36.

10. Victor M, Adams RD, Collins GH. The Wernicke-Korsakoff syndrome. A clinical and pathological study of 245 patients, 82 with post-mortem examinations. Contemp Neurol Ser 1971;7:1-206.

11. Victor M, Adams RD, Collins GH. The Wernicke-Korsakoff syndrome and related neurologic disorders due to alcoholism and malnutrition. 2nd ed. Philadelphia: F.A. Davis; 1989.

12. Day E, Bentham PW, Callaghan R, et al. Thiamine for prevention and treatment of Wernicke-Korsakoff syndrome in people who abuse alcohol. Cochrane Database Syst Rev 2013;(7):CD004033.

13. Day GS, del Campo CM. Wernicke encephalopathy: a medical emergency. CMAf 2014;186:E295

14. Thomson AD, Cook CC, Touquet R, et al.; Royal College of Physicians, London. The Royal College of Physicians report on alcohol: guidelines for managing Wernicke's encephalopathy in the accident and emergency department. Alcobol Alcohol 2002;37:513-21.

15. Galvin R, Brathen G, Ivashynka A, et al. EFNS guidelines for diagnosis, therapy and prevention of Wernicke encephalopathy. Eur 7 Neurol 2010;17: $1408-18$.

16. National Institute for Health and Clinical Excellence. Alcohol-use disorders: diagnosis and clinical management of alcohol-related physical complications. London (UK): National Health Service; 2010, updated 2017.

17. Isenberg-Grzeda E, Chabon B, Nicolson SE. Prescribing thiamine to inpatients with alcohol use disorders: How well are we doing? 7 Addict Med 2014;8:1-5. 
18. Linder LM, Robert S, Mullinax K, et al. Thiamine prescribing and Wernicke's encephalopathy risk factors in patients with alcohol use disorders at a psychiatric hospital. 7 Psychiatr Pract 2018;24:317-22.

19. Alim U, Bates D, Langevin A, et al. Thiamine prescribing practices for adult patients admitted to an internal medicine service. Can 7 Hosp Pharm 2017;70: 179-87.

20. Nakamura ZM, Tatreau JR, Rosenstein DL, et al. Clinical characteristics and outcomes associated with high-dose intravenous thiamine administration in patients with encephalopathy. Psychosomatics 2018;59:379-87.

21. Cravioto H, Korein J, Silberman J. Wernicke's encephalopathy. A clinical and pathological study of 28 autopsied cases. Arch Neurol 1961;4:510-9.

22. Harper C. The incidence of Wernicke's encephalopathy in Australia - a neuropathological study of 131 cases. 7 Neurol Neurosurg Psychiatry 1983;46:593-8.

23. Day E, Callaghan R, Kuruvilla T, et al. Pharmacy-based intervention in Wernicke's encephalopathy. Psychiatrist 2018;34:234-8.

24. Isenberg-Grzeda E, Kutner HE, Nicolson SE. Wernicke-Korsakoff syndrome: under-recognized and under-treated. Psychosomatics 2012;53:507-16.

25. Thomson AD, Marshall EJ, Bell D. Time to act on the inadequate management of Wernicke's encephalopathy in the UK. Alcohol Alcohol 2013;48:4-8.

26. Quinn S, Samuel R, Bolton J, et al. Pharmacological management of alcohol withdrawal in a general hospital. Psychiatr Bull 2018;32:452-4.

27. Wells C, Loshak H. Standardized hospital order sets in acute care: a review of clinical evidence, cost-effectiveness, and guidelines. Ottawa: Canadian Agency for Drugs and Technologies in Health; 2019.

28. Maraganore DM, Frigerio R, Kazmi N, et al. Quality improvement and practice-based research in neurology using the electronic medical record. Neurol Clin Pract 2015;5:419-29.

29. Sechi G, Serra A. Wernicke's encephalopathy: new clinical settings and recent advances in diagnosis and management. Lancet Neurol 2007;6:442-55.

30. Wai JM, Aloezos C, Mowrey WB, et al. Using clinical decision support through the electronic medical record to increase prescribing of high-dose parenteral thiamine in hospitalized patients with alcohol use disorder. 7 Subst Abuse Treat 2019;99:117-23.

31. Ramayya A, Jauhar P. Increasing incidence of Korsakoff's psychosis in the east end of Glasgow. Alcohol Alcohol 1997;32:281-5.

32. Ferguson RK, Soryal IN, Pentland B. Thiamine deficiency in head injury: A missed insult? Alcohol Alcohol 1997;32:493-500.

33. Wrenn KD, Murphy F, Slovis CM. A toxicity study of parenteral thiamine hydrochloride. Ann Emerg Med 1989;18:867-70.

34. Wrenn KD, Slovis CM. Is intravenous thiamine safe? Am 7 Emerg Med 1992; $10: 165$.

35. Thomson A, Guerrini I, Marshall EJ. Incidence of adverse reactions to parenteral thiamine in the treatment of Wernicke's encephalopathy, and recommendations. Alcohol Alcohol 2019;54:609-14.

36. Wilson EC, Stanley G, Mirza Z. The long-term cost to the UK NHS and social services of different durations of IV thiamine (vitamin $B_{1}$ ) for chronic alcohol misusers with symptoms of Wernicke's encephalopathy presenting at the emergency department. Appl Health Econ Health Policy 2016;14:205-15.

37. Warren DK, Zack JE, Mayfield JL, et al. The effect of an education program on the incidence of central venous catheter-associated bloodstream infection in a medical ICU. Chest 2004;126:1612-8.

38. Miller RS, Norris PR, Jenkins JM, et al. Systems initiatives reduce healthcareassociated infections: a study of 22,928 device days in a single trauma unit. 7 Trauma 2010;68:23-31.
39. Kitchlu A, Abdelshaheed T, Tullis E, et al. Gaps in the inpatient management of chronic obstructive pulmonary disease exacerbation and impact of an evidence-based order set. Can Respir 7 2015;22:157-62.

40. Winston AP, Jamieson CP, Madira W, et al. Prevalence of thiamin deficiency in anorexia nervosa. Int 7 Eat Disord 2000;28:451-4.

41. McCormick LM, Buchanan JR, Onwuameze OE, et al. Beyond alcoholism: Wernicke-Korsakoff syndrome in patients with psychiatric disorders. Cogn Behav Neurol 2011;24:209-16.

42. Matheson FI, Smith KLW, Moineddin R, et al. Mental health status and gender as risk factors for onset of physical illness over 10 years. 7 Epidemiol Community Health 2014;68:64-70.

43. Lin S, Leppla IE, Yan H, et al. Prevalence and improvement of Caine-positive Wernicke-Korsakoff syndrome in psychiatric inpatient admissions. Psychosomatics 2020;61:31-8.

Affiliations: Department of Neurology (Day), Mayo Clinic Florida, Jacksonville, Fla.; Krembil Neuroscience Centre (Ladak), University Health Network; Department of Pharmacy (Ladak, del Campo), University Health Network; Division of Neurology (del Campo), University of Toronto, Toronto, Ont.

Contributors: Gregory Day and Safiya Ladak conceived and designed the study and drafted the manuscript. Safiya Ladak was primarily responsible for data acquisition. Gregory Day was primarily responsible for data analysis and interpretation. Martin del Campo approved the study design and assisted with data interpretation. All of the authors revised the manuscript critically for important intellectual content, approved the final version to be published and agreed to be accountable for all aspects of the work.

Funding: Gregory Day is supported by career development award K23AG064029 from the US National Institutes of Health.

Previous presentation: Preliminary data were reported in abstract form at the 2016 annual meeting of the American Academy of Neurology, Apr. 15-21, 2016, Vancouver, BC.

Data sharing: Anonymized data will be made available to qualified researchers on reasonable request addressed to the corresponding author, Gregory Day, day.gregory@mayo.edu.

Acknowledgements: The authors are grateful for the contributions of Ms. Donna Lowe within the Department of Pharmacy and members from the University Health Network Enterprise Data Warehouse and information technology teams, including Richard Baker, who facilitated data collection. The authors thank Dr. Matthew Brier, Department of Neurology, Washington University School of Medicine, for assistance with implementation and interpretation of regression analyses.

Supplemental information: For reviewer comments and the original submission of this manuscript, please see www.cmajopen.ca/content/8/2/ E383/suppl/DC1. 